Hui Liu* and Xin-Hua Lou

\title{
Crystal structure of (2-p-tolylpyrimidine- $\kappa^{2} C, N$ )(triphenylphosphane- $K P$ ) palladium(II), $\mathrm{C}_{29} \mathrm{H}_{24} \mathrm{ClN}_{2} \mathrm{PPd}$
}

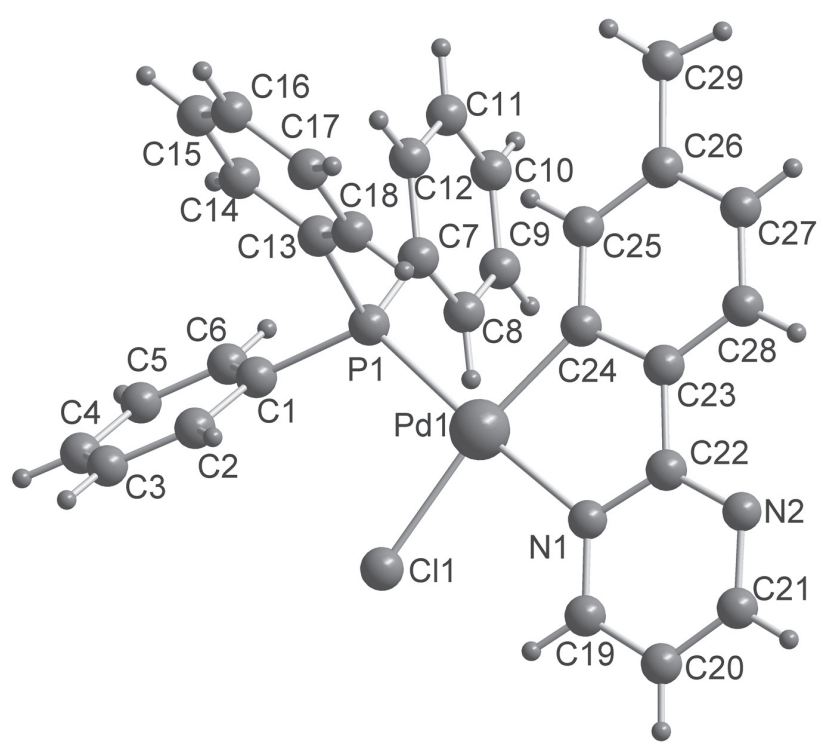

https://doi.org/10.1515/ncrs-2019-0913

Received December 13, 2019; accepted February 5, 2020; available online February 22, 2020

\begin{abstract}
$\mathrm{C}_{29} \mathrm{H}_{24} \mathrm{ClN}_{2} \mathrm{PPd}$, triclinic, $P \overline{1} \quad$ (no. 2), $a=9.9885(8) \AA$, $b=11.5836(8) \AA, \quad c=11.6133(9) \AA, \quad \alpha=76.342(6)^{\circ}$, $\beta=89.821(6)^{\circ}, \quad \gamma=71.435(7)^{\circ}, \quad V=1233.96(16) \AA^{3}, \quad Z=2$, $R_{\text {gt }}(F)=0.0337, w R_{\text {ref }}\left(F^{2}\right)=0.0841, T=291 \mathrm{~K}$.
\end{abstract}

CCDC no.: 1970142

The molecular structure is shown in the figure. Table 1 contains crystallographic data and Table 2 contains the list of the atoms including atomic coordinates and displacement parameters.

\footnotetext{
*Corresponding author: Hui Liu, School of Chemistry and Chemical Engineering, Zhengzhou Normal University, Zhengzhou 450044, P.R. China, e-mail: Ihzznu@163.com. https://orcid.org/0000-00019787-4254

Xin-Hua Lou: College of Food and Pharmacy, Luoyang Normal University, Luoyang 471022, P.R. China
}

๑ Open Access. @ 2020 Hui Liu et al., published by De Gruyter. (cc)BY
Table 1: Data collection and handling.

\begin{tabular}{|c|c|}
\hline Crystal: & Yellow block \\
\hline Size: & $0.20 \times 0.18 \times 0.18 \mathrm{~mm}$ \\
\hline Wavelength: & Cu $K \alpha$ radiation (1.54184 $\AA$ ) \\
\hline$\mu:$ & $7.83 \mathrm{~mm}^{-1}$ \\
\hline Diffractometer, scan mode: & Xcalibur, $\omega$ \\
\hline$\theta_{\max }$, completeness: & $67.1^{\circ},>99 \%$ \\
\hline$N(h k l)_{\text {measured }}, N(h k l)_{\text {unique }}, R_{\text {int }}:$ & $8906,4400,0.031$ \\
\hline Criterion for $I_{\mathrm{obs}}, N(h k l)_{\mathrm{gt}}$ : & $I_{\text {obs }}>2 \sigma\left(I_{\text {obs }}\right), 3959$ \\
\hline$N(\text { param })_{\text {refined }}:$ & 309 \\
\hline Programs: & $\begin{array}{l}\text { CrysAlis }{ }^{\text {PRO }}[1] \text {, Olex2 [2], } \\
\text { SHELX [3] }\end{array}$ \\
\hline
\end{tabular}

\section{Source of material}

The title compound was obtained from the bridge-splitting reaction of the palladacyclic dimer of 2- $p$-tolylpyrimidine and $\mathrm{PPh}_{3}$ according to the literature [4] and recrystallized from a dichloromethane/petroleum ether solution at room temperature to give the desired crystals suitable for single-crystal $\mathrm{X}$-ray diffraction.

\section{Experimental details}

Data collection and reduction were performed using the CrysAlis ${ }^{\mathrm{PRO}}$ software [1]. The structures were solved by direct methods and refined by the least-squares method with Olex2 [2] and SHELXL [3]. All non-hydrogen atoms were refined anisotropically, while hydrogen atoms were placed in geometrically calculated positions and refined using the riding model and appropriate SHELXL AFIX commands.

\section{Comment}

Pyrimidine derivatives are an important class of molecules due to their broad area of application in pharmaceuticals and functional materials [5-7]. In addition, pyrimidines as ligands are also used in organometallic chemistry and catalysis [810]. For example, cyclometalated pyrimidine complexes have been successfully applied to coupling reactions [11-13]. Here we report the crystal structure of the title cyclopalladated 2-p-tolylpyrimidine complex.

The title complex adopts a trans configuration of the coordinated $\mathrm{PPh}_{3}$ ligand to the nitrogen atom of the pyrimidine ligand [14]. The palladium center feature a square planar 
Table 2: Fractional atomic coordinates and isotropic or equivalent isotropic displacement parameters $\left(\AA^{2}\right)$.

\begin{tabular}{|c|c|c|c|c|}
\hline Atom & $x$ & $y$ & $z$ & $U_{\text {iso }}{ }^{*} / U_{\text {eq }}$ \\
\hline Pd1 & $0.75900(3)$ & $0.82567(2)$ & $0.80146(2)$ & $0.03241(10)$ \\
\hline $\mathrm{Cl} 1$ & $0.57819(12)$ & $0.82255(11)$ & $0.93271(11)$ & $0.0602(3)$ \\
\hline $\mathrm{P} 1$ & $0.72495(9)$ & $0.67552(8)$ & $0.72113(8)$ & $0.0320(2)$ \\
\hline N1 & $0.7994(3)$ & $0.9666(3)$ & $0.8685(3)$ & $0.0367(7)$ \\
\hline N2 & $0.9434(4)$ & $1.0973(3)$ & $0.8417(4)$ & $0.0536(9)$ \\
\hline C1 & $0.5891(4)$ & $0.6072(3)$ & $0.7807(3)$ & $0.0352(8)$ \\
\hline $\mathrm{C} 2$ & $0.4498(4)$ & $0.6851(4)$ & $0.7715(4)$ & $0.0480(10)$ \\
\hline $\mathrm{H} 2$ & 0.4277 & 0.7709 & 0.7383 & $0.058^{\star}$ \\
\hline C3 & $0.3428(4)$ & $0.6369(4)$ & $0.8112(4)$ & $0.0537(11)$ \\
\hline H3 & 0.2496 & 0.6902 & 0.8044 & $0.064^{\star}$ \\
\hline $\mathrm{C} 4$ & $0.3740(5)$ & $0.5111(5)$ & $0.8602(4)$ & $0.0591(12)$ \\
\hline H4 & 0.3024 & 0.4785 & 0.8870 & $0.071^{*}$ \\
\hline$C 5$ & $0.5118(5)$ & $0.4329(4)$ & $0.8697(4)$ & $0.0619(12)$ \\
\hline H5 & 0.5331 & 0.3473 & 0.9038 & $0.074^{\star}$ \\
\hline C6 & $0.6188(4)$ & $0.4794(4)$ & $0.8295(4)$ & $0.0472(10)$ \\
\hline H6 & 0.7112 & 0.4249 & 0.8350 & $0.057^{*}$ \\
\hline $\mathrm{C7}$ & $0.8840(4)$ & $0.5371(3)$ & $0.7422(3)$ & $0.0347(8)$ \\
\hline $\mathrm{C} 8$ & $0.9489(4)$ & $0.4875(4)$ & $0.8553(4)$ & $0.0530(11)$ \\
\hline $\mathrm{H} 8$ & 0.9137 & 0.5269 & 0.9154 & $0.064^{*}$ \\
\hline C9 & $1.0662(5)$ & $0.3794(4)$ & $0.8810(4)$ & $0.0574(12)$ \\
\hline H9 & 1.1085 & 0.3461 & 0.9581 & $0.069^{\star}$ \\
\hline $\mathrm{C} 10$ & $1.1194(4)$ & $0.3221(4)$ & $0.7936(4)$ & $0.0537(11)$ \\
\hline $\mathrm{H} 10$ & 1.1984 & 0.2498 & 0.8109 & $0.064^{*}$ \\
\hline C11 & $1.0574(5)$ & $0.3700(4)$ & $0.6808(4)$ & $0.0607(12)$ \\
\hline $\mathrm{H} 11$ & 1.0946 & 0.3307 & 0.6212 & $0.073^{*}$ \\
\hline C12 & $0.9390(5)$ & $0.4775(4)$ & $0.6543(4)$ & $0.0505(10)$ \\
\hline $\mathrm{H} 12$ & 0.8965 & 0.5094 & 0.5771 & $0.061^{*}$ \\
\hline C13 & $0.6650(4)$ & $0.7275(4)$ & $0.5632(3)$ & $0.0374(8)$ \\
\hline C14 & $0.6190(4)$ & $0.6514(4)$ & $0.5078(4)$ & $0.0523(10)$ \\
\hline H14 & 0.6243 & 0.5709 & 0.5501 & $0.063^{*}$ \\
\hline C15 & $0.5656(5)$ & $0.6943(6)$ & $0.3906(4)$ & $0.0671(14)$ \\
\hline H15 & 0.5379 & 0.6417 & 0.3535 & $0.081^{*}$ \\
\hline C16 & $0.5529(5)$ & $0.8150(5)$ & $0.3279(4)$ & $0.0689(15)$ \\
\hline H16 & 0.5149 & 0.8445 & 0.2494 & $0.083^{*}$ \\
\hline C17 & $0.5966(5)$ & $0.8904(5)$ & $0.3819(4)$ & $0.0666(14)$ \\
\hline H17 & 0.5892 & 0.9713 & 0.3395 & $0.080^{\star}$ \\
\hline C18 & $0.6520(4)$ & $0.8485(4)$ & $0.4995(4)$ & $0.0496(10)$ \\
\hline $\mathrm{H} 18$ & 0.6804 & 0.9015 & 0.5355 & $0.060^{*}$ \\
\hline C19 & $0.7340(4)$ & $1.0196(4)$ & $0.9519(4)$ & $0.0477(10)$ \\
\hline H19 & 0.6618 & 0.9939 & 0.9886 & $0.057^{\star}$ \\
\hline $\mathrm{C} 20$ & $0.7720(5)$ & $1.1120(4)$ & $0.9847(4)$ & $0.0558(11)$ \\
\hline $\mathrm{H} 2 \mathrm{O}$ & 0.7285 & 1.1485 & 1.0442 & $0.067^{*}$ \\
\hline C21 & $0.8762(5)$ & $1.1480(4)$ & $0.9261(4)$ & $0.0583(12)$ \\
\hline $\mathrm{H} 21$ & 0.9018 & 1.2116 & 0.9462 & $0.070^{\star}$ \\
\hline $\mathrm{C} 22$ & $0.9032(4)$ & $1.0064(3)$ & $0.8160(4)$ & $0.0419(9)$ \\
\hline C23 & $0.9722(4)$ & $0.9398(3)$ & $0.7286(4)$ & $0.0415(9)$ \\
\hline C24 & $0.9234(4)$ & $0.8449(3)$ & $0.7051(3)$ & $0.0353(8)$ \\
\hline $\mathrm{C} 25$ & $0.9952(4)$ & $0.7810(4)$ & $0.6246(4)$ & $0.0429(9)$ \\
\hline $\mathrm{H} 25$ & 0.9656 & 0.7183 & 0.6070 & $0.051^{*}$ \\
\hline C26 & $1.1100(4)$ & $0.8066(4)$ & $0.5690(4)$ & $0.0477(10)$ \\
\hline C27 & $1.1522(4)$ & $0.9017(4)$ & $0.5915(4)$ & $0.0508(10)$ \\
\hline $\mathrm{H} 27$ & 1.2266 & 0.9213 & 0.5526 & $0.061^{*}$ \\
\hline C28 & $1.0855(4)$ & $0.9681(4)$ & $0.6713(4)$ & $0.0487(10)$ \\
\hline
\end{tabular}

Table 2 (continued)

\begin{tabular}{lrrrr}
\hline Atom & $\boldsymbol{x}$ & $\boldsymbol{y}$ & $\boldsymbol{z}$ & $\boldsymbol{U}_{\text {iso }} \boldsymbol{U}_{\text {eq }}$ \\
\hline H28 & 1.1156 & 1.0314 & 0.6870 & $0.058^{*}$ \\
C29 & $1.1881(5)$ & $0.7302(5)$ & $0.4862(5)$ & $0.0680(14)$ \\
H29A & 1.2263 & 0.6434 & 0.5285 & $0.102^{*}$ \\
H29B & 1.2638 & 0.7601 & 0.4561 & $0.102^{*}$ \\
H29C & 1.1239 & 0.7384 & 0.4211 & $0.102^{*}$ \\
\hline
\end{tabular}

coordination geometry. The $\mathrm{Pd}-\mathrm{P}[2.2707(9) \AA]$ and $\mathrm{Pd}-\mathrm{N}$ [2.110(3) $\AA$ ] bond lengths of the title complex are similar to those of related palladacycles $[8,15-17]$. The chelate ring of the palladacycle Pd1-N1-C22-C23-C24 is virtually planar, with the greatest deviation from the fitted least-squares mean plane being 0.023(3) A for N1. The title complex exists as a dimer in the crystal due to intermolecular hydrogen bonds between chlorine atom and the adjacent $\mathrm{C}-\mathrm{H}$ group of $\mathrm{PPh}_{3}$ $(\mathrm{Cl} \cdots \mathrm{H}=2.886 \AA)$ [17-19].

Acknowledgements: This work was supported by the Key Scientific Research Project Plan of Higher Education Institutions in Henan Province (No. 20A150008), Analytical Chemistry Course Teaching Team of Zhengzhou Normal University (no. 901029) and Environmental Catalytic Scientific Research Innovation Team of Zhengzhou Normal University (702010).

\section{References}

1. Agilent. CrysAlis PRO. Agilent Technologies, Yarnton, Oxfordshire, England (2013).

2. Dolomanov, O. V.; Bourhis, L. J.; Gildea, R. J.; Howard, J. A. K.; Puschmann, H.: OLEX2: a complete structure solution, refinement and analysis program. J. Appl. Crystallogr. 42 (2009) 339-341.

3. Sheldrick, G. M.: A short history of SHELXTL. Acta Crystallogr. A64 (2008) 112-122.

4. Han, X.; Li, H. M.; Xu, C.; Xiao, Z. Q.; Wang, Z. Q.; Fu, W. J.; Hao, X. Q.; Song, M. P.: Water-soluble palladacycles containing hydroxymethyl groups: synthesis, crystal structures and use as catalysts for amination and Suzuki coupling of reactions. Transition Met. Chem. 41 (2016) 403-411.

5. Walker, S. R.; Carter, E. J.; Huff, B. C.; Morris, J. C.: Variolins and related alkaloids. Chem. Rev. 109 (2009) 3080-3098.

6. Li, H. M.; Tu, T. Y.; Han, X.; Wang, Z. Q.; Fu, W. J.; Hao, X. Q.; Song, M. P.; Xu, C.: C-H Monoarylation of naphthylpyrimidines with aryl chlorides catalyzed by a water-soluble ruthenium complex. Synlett 29 (2018) 1729-1734.

7. Lou, X. H.: Crystal structure of 2-(2-(6-methylpyridin-2yl)naphthalen-1-yl)pyrimidine, $\mathrm{C}_{20} \mathrm{H}_{15} \mathrm{~N}_{3}$. Z. Kristallogr. NCS 234 (2019) 1037-1038.

8. Liu, B.; Xia, Q. Q.; Chen, W. Z.: Direct synthesis of iron, cobalt, nickel, and copper complexes of $N$-heterocyclic carbenes by using commercially available metal powders. Angew. Chem. Int. Ed. 48 (2009) 5513-5516. 
9. Xu, C.; Zhang, Y. P.; Wang, Z. Q.; Liang, T.; Fu, W. J.; Hao, X. Q.; Xu, Y.; Ji, B. M.: Synthesis, characterization and crystal structures of monocyclopalladated and biscyclopalladated 1,10bisferrocenylpyrimidine-monophosphine complexes. Inorg. Chim. Acta 365 (2011) 469-472.

10. Achelle, S.; Ramondenc, Y.; Dupas, G.; Ple, N.: Bis-and tris (arylethynyl)pyrimidine oligomers: synthesis and light-emitting properties. Tetrahedron 64 (2008) 2783-2791.

11. Li, H. M.; Xu, C.; Hao, X. Q.; Li, Z.; Wang, Z. Q.; Fu, W. J.; Song, M. P.: Cationic cyclopalladated ferrocenylpyrimidine complex as an efficient catalyst for the synthesis of substituted biarylmethanol by the Suzuki reaction in water. Inorg. Chim. Acta 404 (2013) 236-240.

12. Xu, C.; Dong, X. M.; Wang, Z. Q.; Hao, X. Q.; Li, Z.; Duan, L. M.; Ji, B. M.; Song, M. P.: Triphenylphosphine-cyclometallated iridium(III) pyrimidine complexes: synthesis, crystal structures and application in $\alpha$-alkylation of ketones with alcohols. J. Organomet. Chem. 700 (2012) 214-218.

13. Dupont, J.; Consorti, C. S.; Spencer, J.: The potential of palladacycles: more than just precatalysts. Chem. Rev. 105 (2005) 2527-2571.
14. Calmuschi-Cula, B.; Kalf, I.; Wang, R.; Englert, U.: The first trans-configured cyclopalladated amine. Organometallics 24 (2005) 5491-5493.

15. Li, H. M.; Wang, Z. Q.; Fu, W. J.; Xu, C.: Crystal structures of two luminescent mononuclear and dinuclear cyclopalladated 2(4-ethylphenyl)pyrazine complexes. J. Struct. Chem. 59 (2018) 209-213.

16. Albert, J.; Granell, J.; Zafrilla, J.; Font-Bardia, M.; Solans, X.: The cyclopalladation reaction of 2-phenylaniline revisited. J. Organomet. Chem. 690 (2005) 422-429.

17. Xiao, Z. Q.; Xu, C.; Li, H. M.; Han, X.; Wang, Z. Q.; Fu, W. J.; Hao, X. Q.; Song, M. P.: Two fluorescent cyclopalladated arylpyrazine complexes: synthesis, crystal structures and application in the double Suzuki coupling of $\mathrm{N}$-heteroaryl halides with 1,4-benzenediboronic acid. Transition Met. Chem. 40 (2015) 501-508.

18. Steiner, T.: The hydrogen bond in the solid state. Angew. Chem. Int. Ed. 41 (2002) 48-76.

19. Nangia, A.: Database research in crystal engineering. CrystEngComm 4 (2002) 93-101. 